\title{
RESOURCES RESERVATION IN THE SEGMENT OF OPTICAL NETWORK WITH OBS
}

The growing demands on transmission quality, flexibility and granularity can be achieved by powerful reservation protocols which are an essential part of networks with burst switching. In today's high-speed optical networks there exist a lot of reservation protocols. In this article we present a design of a new reservation protocol called Search\&Compare. This reservation protocol is designed according to the well-known Segment-based Robust Fast Optical Reservation Protocol. Search\&Compare is parallel-segment based and uses parallel link reservation. Our goal is to describe the behavior of reservation protocol in the transmission with setting the priority for incoming bursts with the QoS utilization.

Keywords: Wavelength-division multiplexing, optical burst switching, reservation protocols, services.

\section{Introduction}

At present there is increasing demand for transmission bandwidth for Internet access from users. Providers must design their core networks to satisfy user's claims in the future. To make this possible they must use the multiplexing methods. The different multiplexing techniques are used to meet the increasing demands for transmission capacity and its quality. Currently the most common method of multiplexing to create highspeed full-optical networks is wavelength-division multiplexing (WDM). This technique allows more efficient data transmission via multiple wavelengths transmitting in single optical fiber. The advantage of WDM is the ability to transmit the data with different transmission speed, modulation and format in every single wavelength [1 - 3].

\section{Optical switching}

The networks nodes are very important in high-speed fulloptical networks. Mainly two ways of incoming data switching are used in the nodes: Optical Packet Switching (OPS) and Optical Burst Switching (OBS).

\subsection{Optical Packet Switching}

At present the packet switching is preferred by many telecommunication providers. This is due to its large deployment in the Internet, Wide Area Networks (WAN), Metropolitan
Area Networks (MAN) and Local Area Networks (LAN). Next Generation Networks task will be to unite each type of networks which will work on IP protocol. Therefore, the optical networks working with packet switching are considered for the future. However, this type of network contains various problems, mainly optical memory, because a packet needs to be saved in memory before its routing, until a routing decision is made. It is obvious that minimal bulk of optical memories has to be equal to maximum bulk of packet. However the constant length of packets is considered in many proposals of OPS nodes. For on time adjustment of optical devices during switching, it will be necessary to insert the guard intervals, which will be at the beginning and at the end of the packet information part. OPS nodes typically demand the low level of aggregation at transmission speed over $10 \mathrm{~Gb} . \mathrm{s}^{-1}$. The packet switching time is much shorter than packet transmission time. For the packet length, which is in $\mu \mathrm{s}$, the switching time in the range of ns will be required [4 - 6].

\subsection{Optical Burst Switching}

Optical burst switching is the type of optical switching which combines the best elements of optical packet switching and wavelength routing, while it avoids its insufficiency (see Fig. 1). It provides the mechanism for big amount of bursts data transportation via transparent optical switching network and could serve as bridging technology between existing wavelength routing optical networks and future networks, using optical packet switching [7 - 11].

\footnotetext{
* Michaela Solanska, Miroslav Markovic, Milan Dado

Department of Telecommunications and Multimedia, Faculty of Electrical Engineering, University of Zilina, Slovakia

E-mail: michaela.solanska@fel.uniza.sk
} 
In OBS networks the incoming data are collected into bursts. This is performed by collecting multiple data into the one bigger unit called burst. The burst can have a constant or variable length. Each burst has generated the header, as a necessity to correct the setting of the switching structure in every node, through which the burst will pass. Just before the burst is transported, the control header is sent to inform all the nodes along the road about the burst arrival. The control header is sent through the independent channel which is on different or same wavelength as transferred burst. In case that a control header and burst are transmitted on the same wavelength, we call it in-band signaling. Another case is out-of-band signaling. In each node, the control header is converted by OEO conversion, because of the unavailability of the all optical control and processing circuits. On the basis of the information contained in the control header the setting of switching node structure is made, which is processed in the control circuit. The information contains the time delay between the control header and burst, size of the burst, required output port, incoming wavelength. Then the new header for transmitting burst is generated. This header is again sent from the node ahead of the data burst. This repeats until the data burst arrives to the destination node. The purpose of the destination node is to segment the data burst [7 - 11].

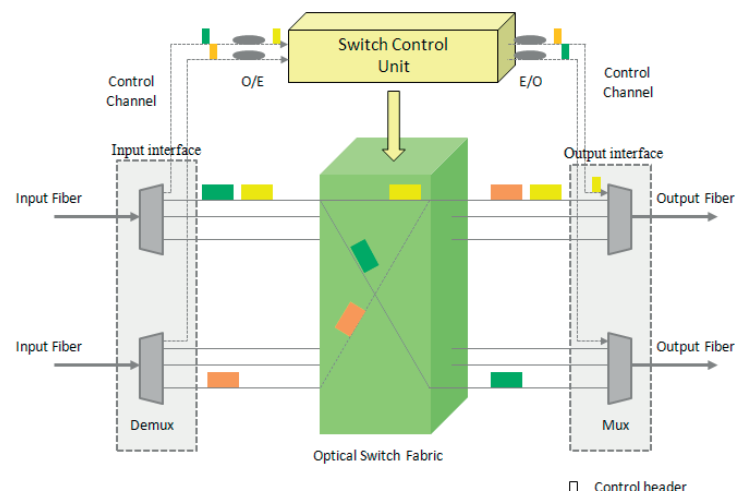

$\square$ Data Burst

Fig. 1 The architecture of $O B S$ node

\subsection{Optical Switches}

Figure 1 shows the architecture of OBS node. The general structure of OBS node consists of five main blocks, both input and output interface, control unit with optical-electrical-optical conversion $(\mathrm{O} / \mathrm{E} / \mathrm{O})$, optical switch (see Table 1 ) and wavelength convertors. Optical switches could be either electronic or optical. In electronic switches it is required to convert incoming data burst and header into the electrical domain, followed by processing and switching to required output port. Only the control header is converted in optical switches. The data burst switching is performed without conversion. Optical switches should meet the following requirements [9 and 10]:

- High switching speed: switching time must be shorter than the one required for burst transmission, i.e. ones to tens $\mu$,

- Dimension of switching structure,

- Low insertion losses or sufficient amplification,

- Low crosstalk between channels,

- Simplicity and easy integrability.

\section{Reservation Protocols}

Reservation protocols are important part of OBS networks. The main reason of reservation protocols in OBS nodes is to reserve their resources for some time period, which is needed for burst transmission. Recently, there are some variations of reservation protocols designed, which are trying to use the resources of OBS nodes most efficiently with the lowest blocking possibility. The best known reservation protocols are: Segmentbased Robust Fast Optical Reservation Protocol (S-RFORP), Robust Fast Optical Reservation Protocol (RFORP), Resource Reservation Protocol-Traffic Engineering (RSVP-TE) and Intermediate-node Initiated Reservation (IIR) [4 and 10].

The suggested reservation protocol Search\&Compare (S\&C) is based on S-RFORP reservation protocol, because it has the best features of the mentioned reservation protocols. It uses S-RFORP to minimize the probability of blocking the parallel amongsegment discovery and reservation, and simultaneously stock inter-segment discovery and reservation. Reservation protocol $\mathrm{S} \& \mathrm{C}$ is an improved version of S-RFORP protocol. Unlike the S-RFORP, it uses parallel inter-segment discovery and reservation through which is possible to achieve less time of inter-segment discovery and reservation [4 and 10].

The reservation protocol S\&C uses out-of-band signaling and its advantage is the possibility to setup the priority for each incoming data burst into the segment due to which it utilizes the QoS. If OBS nodes in networks do not have enough resources for transmitting the required burst, it is necessary to prioritize the bursts based on the recognition of the burst priority for transmission.

\subsection{Reservation of network resources in the segment}

Reservation depending on the type of transmitted services is executed through segments in which the data of different types are transmitted. A segment contains an input edge node which, in this case, simply gathers incoming data (packets) that lead to the same destination node. In the input edge node a wavelength is reserved for each burst which can be the same through the whole burst transmission in OBS network (see Fig. 2). 


\begin{tabular}{|c|c|c|c|c|c|}
\hline \multirow[b]{2}{*}{ Technology } & \multicolumn{5}{|c|}{ Properties } \\
\hline & Dimension & Switching time & $\begin{array}{c}\text { Insertion loss } \\
\text { [dB] }\end{array}$ & $\begin{array}{c}\text { Crosstalk } \\
{[\mathrm{dB}]}\end{array}$ & $\begin{array}{l}\text { PDL loss } \\
\text { [dB] }\end{array}$ \\
\hline Optomechanical & $16 \times 16$ & $<10 \mathrm{~ms}$ & 3 & $<-55$ & 0.2 \\
\hline 2D MEMS & $32 \times 32$ & $<10 \mathrm{~ms}$ & $1.7-6.9$ & $<-60$ & $0.11-0.16$ \\
\hline 3D MEMS & $\begin{array}{l}350 \times 350 \\
160 \times 160\end{array}$ & $\begin{array}{l}<10 \mathrm{~ms} \\
<10 \mathrm{~ms}\end{array}$ & $\begin{array}{c}6 \pm 1 \\
<2\end{array}$ & $\begin{array}{l}<-60 \\
<-55\end{array}$ & $\begin{array}{l}0.4 \\
0.5\end{array}$ \\
\hline $\begin{array}{l}\text { Thermooptical } \\
\text { Silica } \\
\text { Polymer }\end{array}$ & $\begin{array}{c}8 \times 8 \\
16 \times 16\end{array}$ & $\begin{array}{l}<10 \mathrm{~ms} \\
<10 \mathrm{~ms}\end{array}$ & $\begin{array}{l}8 \\
6\end{array}$ & $\begin{array}{l}<-35 \\
<-30\end{array}$ & $\begin{array}{l}0.5 \\
0.4\end{array}$ \\
\hline Liquid - Crystal & $2 \times 2$ & $<10 \mathrm{~ms}$ & 1.5 & $<-35$ & 0.1 \\
\hline Acoustooptical & $1 \times \mathrm{N}$ & $<3 \mu \mathrm{s}$ & 6 & $<-35$ & \\
\hline $\begin{array}{c}\text { Electrooptical } \\
\mathrm{LiNbO}_{3} \\
\mathrm{InP}^{2}\end{array}$ & $\begin{array}{l}8 \times 8 \\
1 \times 2\end{array}$ & $\begin{array}{l}<10 \mathrm{~ns} \\
<10 \mathrm{~ns}\end{array}$ & 9 & $\begin{array}{l}<-35 \\
<-25\end{array}$ & 0.5 \\
\hline SOA & & $1 \mathrm{~ns}$ & $\sim 0$ & $<-50$ & $<1$ \\
\hline
\end{tabular}

In the case of unavailability of the spectral channel with distinct wavelength, on which the burst is transmitted, the node will choose another appropriate wavelength. In the output node the burst is disassembled to data (packets) which are further processed [4 and 7].

To verify the proposed solution, we made the numerical computer model in VPIphotonics environment (VPI). As shown in Fig. 3, to develop one segment, the connection between optical switches had to be proposed and the verification bits of single switches had to be set. The proposed model contains five optical transmitters with RZ modulation, multiplexor, demultiplexor, six optical switches and delay segments.

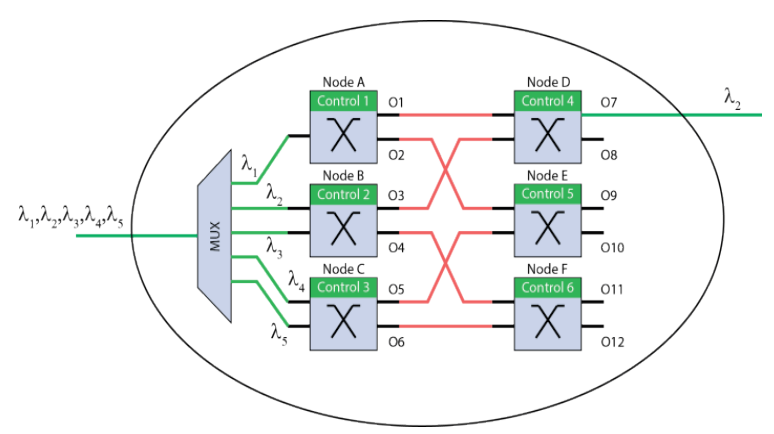

Fig. 2 Transmission depending on the type of transmitted services within one segment

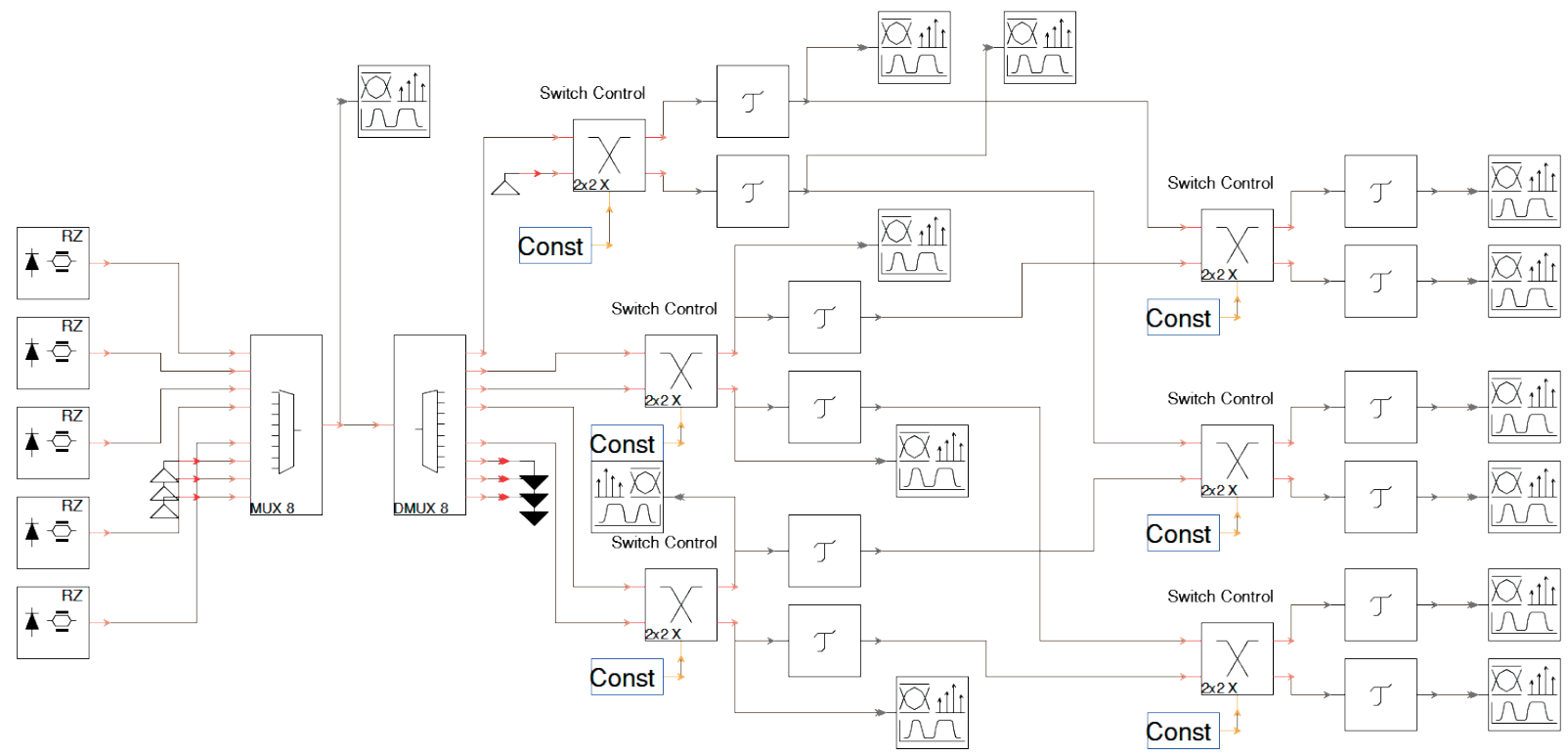

Fig. 3 Scheme of five-channel system in simulation software VPI 


\begin{tabular}{|c|c|c|c|c|c|c|c|}
\hline Variant 1 & & 01 & 02 & 03 & 04 & 05 & 06 \\
\hline Control 1 & 0 & $\lambda_{1}$ & - & $\lambda_{2}$ & $\lambda_{3}$ & $\lambda_{4}$ & $\lambda_{5}$ \\
\hline Control 2 & 0 & $\lambda_{1}$ & - & $\lambda_{2}$ & $\lambda_{3}$ & $\lambda_{4}$ & $\lambda_{5}$ \\
\hline \multirow[t]{2}{*}{ Control 3} & 0 & $\lambda_{1}$ & - & $\lambda_{2}$ & $\lambda_{3}$ & $\lambda_{4}$ & $\lambda_{5}$ \\
\hline & & 07 & 08 & 09 & 010 & 011 & 012 \\
\hline Control 4 & 0 & $\lambda_{1}$ & $\lambda_{2}$ & - & $\lambda_{4}$ & $\lambda_{3}$ & $\lambda_{5}$ \\
\hline Control 5 & 0 & $\lambda_{1}$ & $\lambda_{2}$ & - & $\lambda_{4}$ & $\lambda_{3}$ & $\lambda_{5}$ \\
\hline Control 6 & 0 & $\lambda_{1}$ & $\lambda_{2}$ & - & $\lambda_{4}$ & $\lambda_{3}$ & $\lambda_{5}$ \\
\hline Variant 2 & & 01 & 02 & 03 & 04 & 05 & 06 \\
\hline Control 1 & 1 & - & $\lambda_{1}$ & $\lambda_{2}$ & $\lambda_{3}$ & $\lambda_{5}$ & $\lambda_{4}$ \\
\hline Control 2 & 0 & - & $\lambda_{1}$ & $\lambda_{2}$ & $\lambda_{3}$ & $\lambda_{5}$ & $\lambda_{4}$ \\
\hline \multirow[t]{2}{*}{ Control 3} & 1 & - & $\lambda_{1}$ & $\lambda_{2}$ & $\lambda_{3}$ & $\lambda_{5}$ & $\lambda_{4}$ \\
\hline & & 07 & 08 & O9 & $\mathrm{O} 10$ & O11 & $\mathrm{O} 12$ \\
\hline Control 4 & 1 & $\lambda_{2}$ & - & $\lambda_{1}$ & $\lambda_{5}$ & $\lambda_{4}$ & $\lambda_{3}$ \\
\hline Control 5 & 0 & $\lambda_{2}$ & - & $\lambda_{1}$ & $\lambda_{5}$ & $\lambda_{4}$ & $\lambda_{3}$ \\
\hline Control 6 & 1 & $\lambda_{2}$ & - & $\lambda_{1}$ & $\lambda_{5}$ & $\lambda_{4}$ & $\lambda_{3}$ \\
\hline
\end{tabular}

To control optical switches, the verification bit was chosen, which is shown in Fig. 3 as Const. This constant can be changed to 1 or 0 . Since the ideal optical switch was chosen, it was necessary to add the delay pad (segment) to each switch, which simulates the necessary time to switch the OXC. To illustrate the output signals, an optical signal analyzer was chosen.

By the control bits it is possible to set the switching structure of the switch. In case the control bit is set to 0 , the input ports will not switch, i. e. input 1 will be transferred to the output port, input port 2 will be transferred to output port 2. If the control bit is set to $\lambda$, input port 1 will be switched to output port 2 and input port 2 will be transferred to output port 1. Ports O1-O6 represent the output ports from the first three optical switches, ports $07-012$ represent the output ports of output optical switches. For video transmission the $1_{2}$ wavelength was chosen, which was necessary to transmit from the input edge node to output node of the given segment.

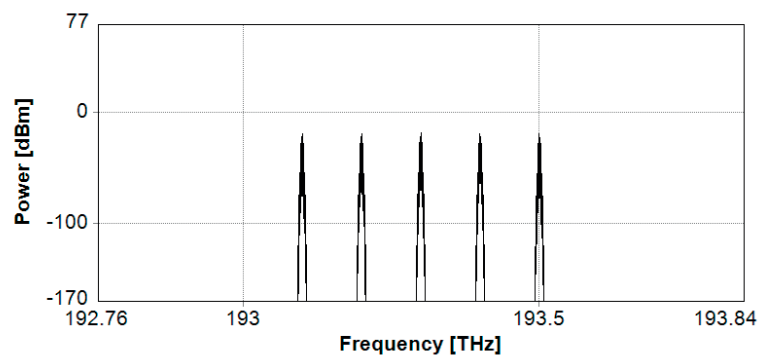

Fig. 4 Optical spectrum of a five-channel system

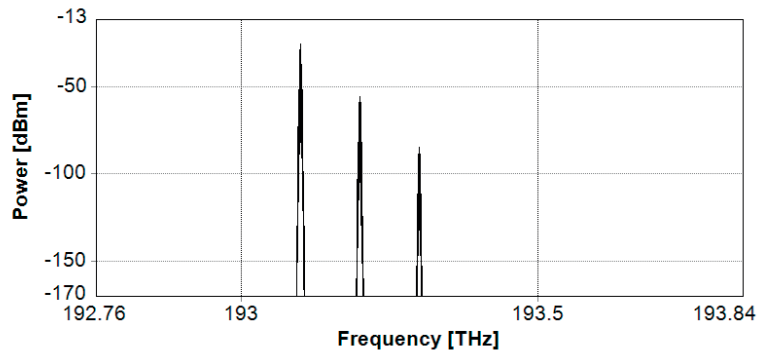

Fig. 5 Optical spectrum on output port 07

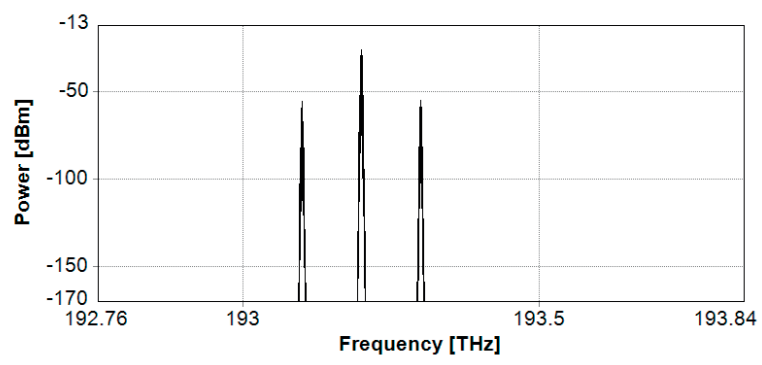

Fig. 6 Optical spectrum on output port 08

Figure 4 shows the optical spectrum of a five-channel system with wavelengths $\lambda_{1}-\lambda_{5}$.

For combination of the control bits 101101 the required wavelength $\lambda_{2}$ will get on output port $\mathrm{O} 7$ (as shown in Fig. 5 and in Table 2). If we need the required wavelength $\lambda_{2}$ on a different 
output port, e.g. on $\mathrm{O} 8$ (see the Fig. 6) as the combination of control bits we will choose 000000 .

\section{Conclusion}

In this document we focused on the development of the numerical computer model of reservation of the resources in one segment. We developed the simulation model in VPIphotonics environment. In simulation model the input parameters were wavelengths of optical transmitters and insertion losses of multiplexor and demultiplexor. Since the optical switch was ideal, it was necessary to add the delay pad to each switch, which simulates the necessary time to switch the OXC. The switching time was set according to Table 1 . The output of the simulation was described in Chapter 3. Our next goal will be the development of the numerical computer model of the optical network which will consist of multiple segments.

\section{Acknowledgements}

This work is supported by the Slovak Research and Development Agency under the project APVV-0025-12 ("Mitigation of stochastic effects in high-bit rate all-optical networks") and the European Regional Development Fund and the Slovak state budget for the project "Research Centre of University of Zilina”, ITMS 26220220183.

\section{References}

[1] SIVAlingAM, K. M., SUBRAMANIAM, S.: Optical WDM Networks: Principles and Practice, Massachusetts : Kluwer Academic, 2000.

[2] SICHANI, A. V., MOUfTAH, H. T. A.: Novel Progressive Reservation Protocol for WDM All-optical Networks. IEEE Intern. Conference on Communications, ICC 2003, 1463-1467, 2003.

[3] BENEDIKOVIC, D., LITVIK, J., KUBA, M., DADO, M., DUBOVAN, J.: Influence of Nonlinear Effects in WDM System with Non-equidistant Channel Spacing using Different Types of High-order PSK and QAM Modulation Formats. Optical Modelling and Design II, SPIE, vol. 8429, 2012.

[4] MARKOVIC, M.: Reservation Protocols in High-speed All-optical Networks with Burst Switching, Dissertation thesis, 2010, University of Zilina.

[5] POBORIL, R., LATAL, J., KOUDELKA, P., VITASEK, J., SISKA, P., SKAPA, J., VASINEK, V.: A Concept of a Hybrid WDM/ TDM Topology using the Fabry-Perot Laser in the Optiwave Simulation Environment, Advances in Electrical and Electronic Engineering, vol. 9, 2011.

[6] MEI, Z., ZHAO, D., LIN, J. A.: Survey of Optical Networks and its Visual Management and Control, $3^{\text {rd }}$ Intern. Conference on Advanced Computer Control, ICACC 2011, 473- 477, 2011.

[7] XU, H., YU, O., YIN, L., LIAO, M. Segment-based Robust Fast Optical Reservation Protocol, High-Speed Networks Workshop, 36-40, 2007.

[8] SALEH, A. M. M, SIMMONS, J. M.: All-optical Networking-evolution, Benefits, Challenges, and Future Vision, IEEE J. \& Magazines, vol. 100, No. 5, 2012.

[9] O’MAHONY, M. J., POLITI, CH., KLONIDIS, D., NEJABITI, R., SIMEONIDOU, D.: Future Optical Networks, J. of Lightwave Technology, vol. 24, No. 12, 2006.

[10] MARKOVIC, M., DUBOVAN, J., DADO, M.: Search-\&-Compare (S\&C) - Reservation Protocol in High-Speed Optical Networks, Electronics and Electrical Engineering, vol. 114, No. 8, 2011.

[11] PAPADIMITRIOU, G., PAPAZOGLOU, CH, POMPORTSIS, A.: Optical Switching, Hoboken : Wiley - Interscience, 2007. 